\title{
Genomic and immunohistochemical characterisation of a lacrimal gland oncocytoma and review of literature
}

\author{
LAUGE HJORTH MIKKELSEN ${ }^{1,2}$, SIMON ANDREASEN ${ }^{3,4}$, LINEA CECILIE MELCHIOR ${ }^{1}$, \\ MARTA PERSSON $^{5}$, JEPPE DYRBERG ANDERSEN $^{6}$, VANIA PEREIRA ${ }^{6}$, PETER BJERRE TOFT $^{2}$, \\ NIELS MORLING ${ }^{6}$, GÖRAN STENMAN ${ }^{5}$ and STEFFEN HEEGAARD ${ }^{1,2}$
}

\author{
Departments of ${ }^{1}$ Pathology and ${ }^{2}$ Ophthalmology, Rigshospitalet, Copenhagen University Hospital, 2100 Copenhagen; \\ ${ }^{3}$ Department of Otorhinolaryngology and Maxillofacial Surgery, Zealand University Hospital, $4600 \mathrm{~K} ø \mathrm{ge} ;{ }^{4}$ Department of \\ Otorhinolaryngology, Head and Neck Surgery and Audiology, Rigshospitalet, Copenhagen University Hospital, \\ 2100 Copenhagen, Denmark; ${ }^{5}$ Department of Pathology and Genetics, Sahlgrenska Cancer Center, University of \\ Gothenburg, 40530 Gothenburg, Sweden; ${ }^{6}$ Section of Forensic Genetics, Department of Forensic Medicine, \\ University of Copenhagen, 2100 Copenhagen, Denmark
}

Received March 16, 2017; Accepted June 21, 2017

DOI: $10.3892 / 01.2017 .6713$

\begin{abstract}
The aim of the present study was to report the genetic and immunohistochemical profile of a rare case of lacrimal gland oncocytoma. A 20-year-old male underwent magnetic resonance imaging (MRI) due to viral encephalitis. Notably, the MRI revealed a multicystic tumor in the left lacrimal gland. A lateral orbitotomy was performed and the tumor was completely excised. Four months following surgery, the patient was free of symptoms. Histopathologically, the tumor was composed of large, eosinophilic and polyhedral cells with small round nuclei. The tumor cells stained strongly for antimitochondrial antibody MU213-UC, cytokeratin (CK) 5/6, CK 7, CK 17, CK 8/18 and CK 19. The final diagnosis was an oncocytoma of the lacrimal gland without any signs of malignancy. Array-based comparative genomic hybridisation demonstrated a gain of one copy of chromosome 8 and loss of one copy of chromosome 22 as the sole genomic imbalances. These chromosomal alterations have not previously been identified in oncocytoma and may be specific to lacrimal gland oncocytoma. Sequencing of the mitochondrial genome demonstrated multiple alterations of the NADH-ubiquinone oxidoreductase chain 5 (ND5) gene involved in mitochondrial oxidative phosphorylation. This may support the notion of a common genetic background of oncocytic lesions in the lacrimal gland and other anatomical sites.
\end{abstract}

Correspondence to: Professor Steffen Heegaard, Department of Pathology, Rigshospitalet, Copenhagen University Hospital, Frederik V's Vej 11, 2100 Copenhagen, Denmark

E-mail: sthe@sund.ku.dk

Key words: oncocytoma, lacrimal gland, oncocytic, mitochondrial DNA, genetics

\section{Introduction}

Oncocytoma is a rare benign neoplasm that may arise in the glandular epithelium of salivary glands, thyroid, parathyroid, kidney, adrenal cortex, and in the ocular region (1-3). The oncocytic cells (oncocytes, from the Greek onkoustai, meaning to swell) have a swollen appearance, and are characterised by a large number of morphologically abnormal and possibly dysfunctional mitochondria $(2,4)$. Ocular adnexal oncocytoma is rare and occurs most frequently in the caruncle, although lesions have also been identified on the eyelid margin, the conjunctiva, in the lacrimal sac, and in the lacrimal gland (1). In 1959, the first case of a lacrimal gland oncocytoma was reported by Beskid and Zarzycka (5), since then 12 additional cases have been reported (Table I) (5-16). The molecular pathogenesis of lacrimal gland oncocytoma, and the mechanisms leading to the combined intracellular mitochondrial proliferation and the proliferation of the oncocyte itself remains unclear (2). It has been suggested that mitochondria may be involved in oncocytoma pathogenesis, and several studies have identified multiple sequence variants in mitochondrial DNA (mtDNA), particularly in genes involved in the cellular respiration complex I (2,4,16-18). In the present study, a case of lacrimal gland oncocytoma was described, and a detailed immunohistochemical profile was presented along with a genetic profile of the tumor based on high-resolution array comparative genomic hybridisation (aCGH) of nuclear DNA and next-generation sequencing of the mitochondrial genome.

\section{Materials and methods}

Clinical history. A 20-year-old male was hospitalised from January 3rd to January 21st 2015 (Rigshospitalet, Copenhagen University Hospital, Denmark) due to encephalitic symptoms. Unexpectedly, magnetic resonance imaging revealed an $\sim 2 \times 2 \times 2 \mathrm{~cm}$ multicystic tumor in the lacrimal gland of the left orbit along with a $5-\mathrm{mm}$ protrusion of the left eye causing 


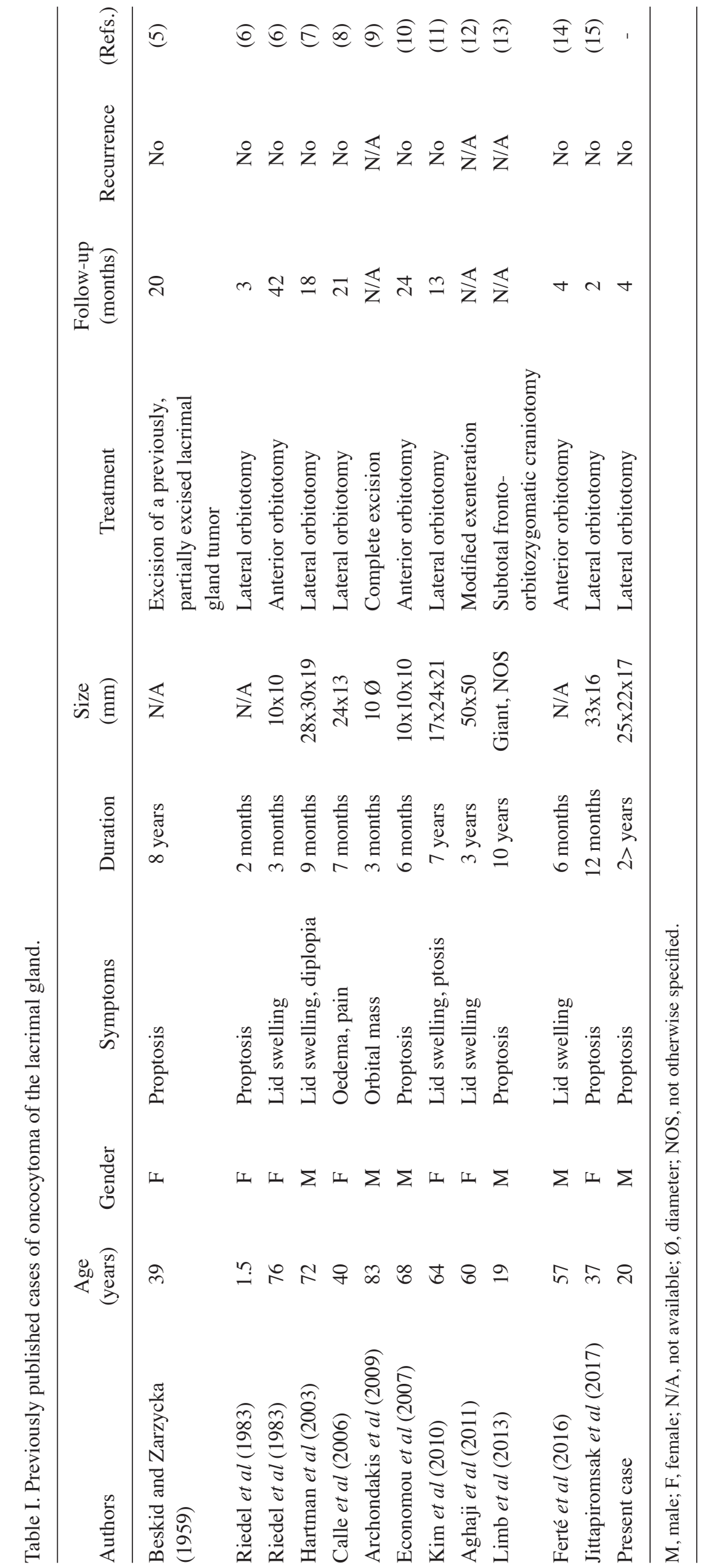




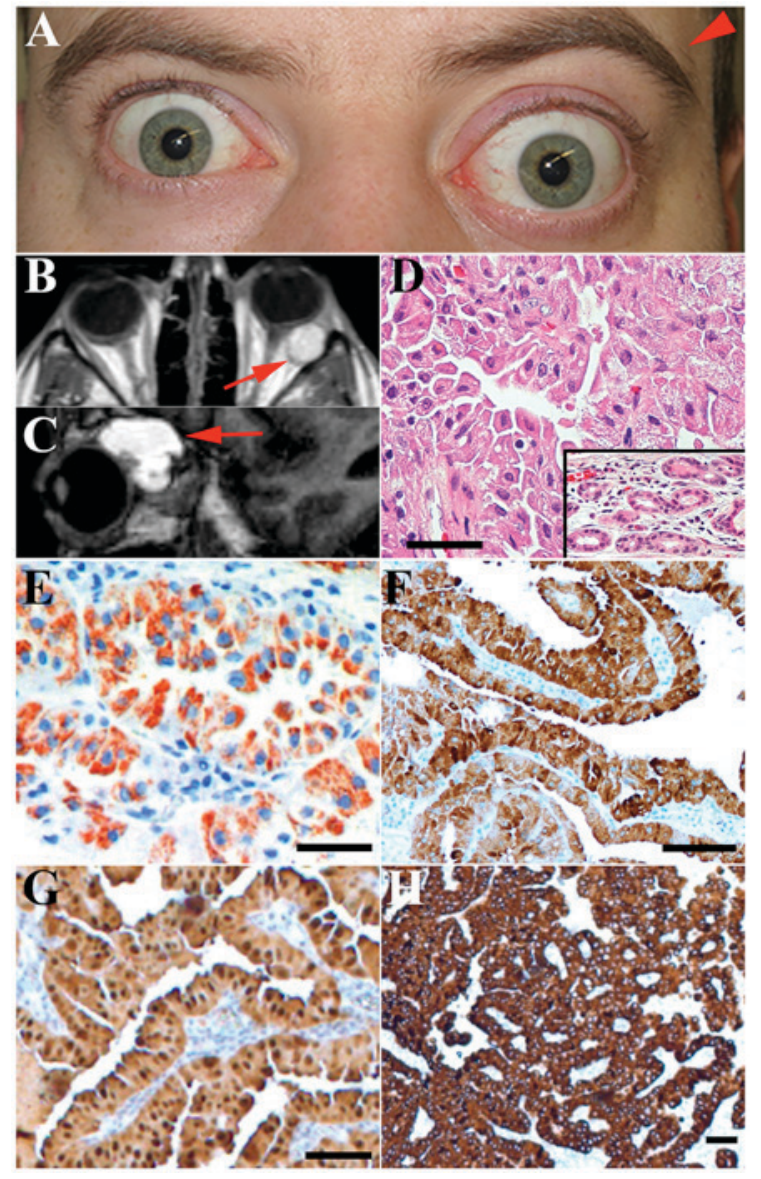

Figure 1. (A) A 20-year-old male presented with left-sided proptosis as the only finding (arrow), the bilateral upper lid retraction was habitual. (B) Axial and (C) sagittal magnetic resonance imaging scans demonstrating a cystic lacrimal gland tumor measuring $2 \times 2 \times 2 \mathrm{~cm}$ in the left orbit (arrowheads). The tumor was located far posteriorly for a lacrimal gland tumor. This was due to a cystic tumor area expanding posteriorly. (D) The tumor cells were large and eosinophilic with abundant granular cytoplasm (haematoxylin and eosin bar, $50 \mu \mathrm{m}$ ). Inset presents normal lacrimal gland tissue in the periphery of the specimen. (E) The tumor cells contained abundant mitochondria (anti-MU213-UC staining, red; bar, $50 \mu \mathrm{m}$ ). (F) The tumor cells stained positively for EMA (bar, $150 \mu \mathrm{m}$ ). (G) The tumor cells stained positively for S-100 (bar, $100 \mu \mathrm{m}$ ). (H) The tumor cells stained positively for CK8 (bar, $50 \mu \mathrm{m})$.

pronounced asymmetry of the orbital region (Fig. 1A-C). Computer tomography confirmed that the orbital roof was intact without tumor infiltration. The orbital roof was remodelled consistent with a slow growing benign tumor. The tumor expanded posteriorly in the orbit due to cystic areas in the tumor. The patient received treatment for encephalitis, and was subsequently referred for ophthalmic evaluation of the lacrimal gland mass. No visual symptoms, pain, or cosmetic changes had been noticed by the patient. Images revealed that the patient had symmetry of the orbital region two years previously. On examination prior to operation, visual acuity measured with the Snellen chart was normal (6/6 s.c.) in the right eye and reduced (6/30 s.c.) in the left eye. The patient was not amblyopic and the reduced visual acuity in the left eye was explained by the refraction error caused by the tumor mass deforming the eyeball (Fig. 1B). Proptosis (Hertel 18/23-95) of the left eye was present, and the eye was displaced $2 \mathrm{~mm}$ medially and downwards. When examined the patient reported vertical diplopia. The intraocular pressure was $12 \mathrm{mmHg}$ in the right eye and $15 \mathrm{mmHg}$ in the left eye measured with Goldman applanation tonometry. Palpation of the lacrimal fossa revealed a smooth mass. The patient had decreased superolateral movement of the left eye due to the space-occupying lesion. Slit-lamp microscopy, including ophthalmoscopy was normal. Pupillary reflexes, colour vision and visual fields were normal. A lateral orbitotomy was performed, and the tumor was completely excised. Four months following surgery, the visual acuity was $6 / 6$ s.c. in both eyes and the patient was free of any symptoms.

Histopathology and immunohistochemistry. Formalin-fixed paraffin-embedded (FFPE) tissue from the resected orbital tumor was sectioned and stained with haematoxylin and eosin, Alcian blue, periodic acid-Schiff (PAS), and phosphotungstic acid-haematoxylin (PTAH) according to standard protocols as previously described (1). Immunohistochemical stainings of $4 \mu \mathrm{m}$ sections were performed using the following antibodies: Mitochondrial antibody MU213-UC (monoclonal, clone no. 113-1; cat no. MU2130506; mouse anti-human; 1:10; BioGenex Laboratories, Inc., San Ramon, CA, USA), Ki-67 (monoclonal, clone MIB-1, cat no. M724001, mouse anti-human; 1:100), S-100 (polyclonal, cat no. Z0311, rabbit anti-human; 1:4,000), cytokeratin (CK) 5/6 (monoclonal, clone D5/16 B4, cat no. M723701, mouse anti-human; 1:20), CK 7 (monoclonal, clone OV-TL 12/30, cat no. M701801, mouse anti-human; 1:1,000), CK 8/18 (monoclonal, clone EP17/EP30, cat no. M365201-2, rabbit anti-human; 1:50) (all from Dako; Agilent Technologies, Inc., Santa Clara, CA, USA), CK 14 (monoclonal, clone LL002, cat no. NCL-LL002; mouse anti-human; 1:40; Novocastra; Leica Biosystems Newcastle, Newcastle, UK), CK 17 (monoclonal, clone E3, cat no. M 7046; mouse anti-human; 1:20), CK 19 (monoclonal, clone RCK 108, cat no. M0888; mouse anti-human; 1:100), CK 20 (monoclonal, clone Ks 20.8, cat no. M 7019; mouse anti-human), cluster of differentiation (CD)117 (polyclonal, cat no. A450229, rabbit anti-human; 1:100), smooth muscle actin (SMA; monoclonal, clone 1A4, cat no. M0851, mouse anti-human, 1:100), carcinoembryonic antigen (CEA; monoclonal, clone IL-7, cat no. M7072; mouse anti-human; 1:50), and epithelial membrane antigen (EMA; monoclonal, clone E29, cat no. M0613; mouse antihuman; 1:1,000) (all from Dako; Agilent Technologies, Inc.). The blocking reagent was peroxidase $\left(37^{\circ} \mathrm{C}, 10 \mathrm{~min}\right.$ incubation). Incubation with primary antibody occurred at $37^{\circ} \mathrm{C}$ for $32 \mathrm{~min}$. Secondary antibodies were used as previously described (1). Immunohistochemistry was performed using the Ventana BenchMark ULTRA platform (Ventana Medical Systems, Inc., Tucson, AZ, USA). The MU213-UC staining was performed using a biotin-free method (EnVision Flex+; Dako; Agilent Technologies, Inc.) to avoid a false-positive reaction caused by endogenous biotin in the mitochondrial-rich tissue as previously described (1). Appropriate controls were included. All slides were assessed using an Axioplan 2 Imaging light microscope (Zeiss, Oberkochen, Germany). The investigation adheres to the tenets of the Declaration of Helsinki (19) and the patient provided informed consent.

$a C G H$. Genomic DNA was isolated from FFPE tumor tissue using the QIAamp ${ }^{\circledR}$ DNA FFPE Tissue kit (Qiagen $\mathrm{GmbH}$, 
Table II. Mitochondrial DNA sequencing.

\begin{tabular}{|c|c|c|c|c|c|}
\hline SNP & MT-locus & Amino acid change & Frequency & GenBank frequency $(\%)$ & Polyphen-2.2 (score) \\
\hline A73G & D-loop & Non-coding & 100.0 & $23631(73.71)$ & \\
\hline C150T & D-loop & Non-coding & 98.3 & $3787(11.81)$ & \\
\hline G185A & D-loop & Non-coding & 100.0 & $1274(3.97)$ & \\
\hline A263G & D-loop & Non-coding & 100.0 & $29979(93.51)$ & \\
\hline 323_324insC & D-loop & Non-coding & 100.0 & $0(0.00)$ & \\
\hline A750G & RNR1 (12s-RNA) & rRNA & 100.0 & $31410(97.98)$ & \\
\hline A1438G & RNR1 (12s-RNA) & rRNA & 100.0 & $30179(94.14)$ & \\
\hline $\mathrm{C} 1721 \mathrm{~T}$ & RNR2 (16s-RNA) & rRNA & 100.0 & $225(0.70)$ & \\
\hline A2706G & RNR2 (16s-RNA) & rRNA & 100.0 & $24784(77.31)$ & \\
\hline T3197C & RNR2 (16s-RNA) & rRNA & 100.0 & $1350(4.21)$ & \\
\hline $\mathrm{A} 3243 \mathrm{G}^{\mathrm{a}}$ & L(UUA/G)/TER & tRNA & 94.5 & $8(0.02)$ & \\
\hline A4769G & ND2 & Synonymous & 100.0 & $31182(97.26)$ & \\
\hline $\mathrm{A} 4958 \mathrm{G}^{\mathrm{a}}$ & ND2 & Synonymous & 100.0 & $115(0.36)$ & \\
\hline C7028T & $\mathrm{CO} 1$ & Synonymous & 100.0 & $25290(78.89)$ & \\
\hline A7768G & $\mathrm{CO} 2$ & Synonymous & 100.0 & $592(1.85)$ & \\
\hline A8860G & ATP6 & Non-syn:Thr $\rightarrow$ Ala & 100.0 & 31527 (98.34) & Benign (0) \\
\hline G9477A & $\mathrm{CO} 3$ & Non-syn:Val $\rightarrow$ Ile & 100.0 & 1344 (4.19) & Benign (0) \\
\hline A9670G ${ }^{\mathrm{a}}$ & $\mathrm{CO} 3$ & Non-syn: Asn $\rightarrow$ Ser & 100.0 & $28(0.09)$ & Benign (0.01) \\
\hline A11467G & ND4 & Synonymous & 100.0 & $4213(13.14)$ & \\
\hline A11653G & ND4 & Synonymous & 100.0 & $194(0.61)$ & \\
\hline G11719A & ND4 & Synonymous & 100.0 & $24160(75.36)$ & \\
\hline A12308G & $\mathrm{L}(\mathrm{CUN})$ & tRNA & 97.5 & $4193(13.08)$ & \\
\hline G12372A & ND5 & Synonymous & 100.0 & $4519(14.10)$ & \\
\hline $\mathrm{A} 12530 \mathrm{G}^{\mathrm{a}}$ & ND5 & Non-syn:Asn $\rightarrow$ Ser & 100.0 & $20(0.06)$ & Benign (0.06) \\
\hline $\mathrm{A} 12634 \mathrm{G}^{\mathrm{a}}$ & ND5 & Non-syn:Ile $\rightarrow$ Val & 100.0 & $91(0.28)$ & Probably damaging (1) \\
\hline T13617C & ND5 & Synonymous & 100.0 & $1315(4.10)$ & \\
\hline $\mathrm{A} 13630 \mathrm{G}^{\mathrm{a}}$ & ND5 & Non-syn:Thr $\rightarrow$ Ala & 100.0 & $62(0.19)$ & Benign (0.05) \\
\hline A13637G & ND5 & Non-syn:Gln $\rightarrow$ Arg & 100.0 & $287(0.90)$ & Benign (0.1) \\
\hline $\mathrm{T} 14182 \mathrm{C}$ & ND6 & Synonymous & 100.0 & $843(2.63)$ & \\
\hline $\mathrm{C} 14766 \mathrm{~T}$ & CYTB & Non-syn:Thr $\rightarrow$ Ile & 100.0 & $24091(75.15)$ & Benign (0.01) \\
\hline A15326G & CYTB & Non-syn:Thr $\rightarrow$ Ala & 100.0 & 31512 (98.29) & Benign (0.02) \\
\hline $\mathrm{C} 16270 \mathrm{~T}$ & D-loop & Non-coding & 100.0 & $8147(25.41)$ & \\
\hline T16519C & D-loop & Non-coding & 100.0 & $1694(5.28)$ & \\
\hline
\end{tabular}

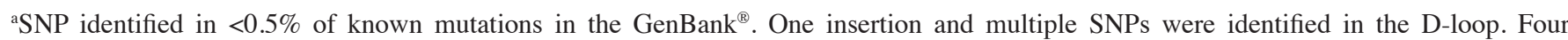
non-synonymous (non-syn) SNPs were identified in the ND5 region, which is a part of the respiratory complex I. The Polyphen-2 tool predicts the potential damage provided by an SNP. MT, mitochondrial; SNP, Single nucleotide polymorphism; ND, NADH-ubiquinone oxidoreductase chain; D-loop, displacement-loop.

Hilden, Germany) according to the manufacturer's protocol. aCGH analysis was performed using the $180 \mathrm{~K}$ oligonucleotide CGH microarray (G4449A; Agilent Technologies Inc.) as previously described $(20,21)$. The slide was scanned on an Agilent High-Resolution C Microarray scanner, followed by data extraction and normalisation using Feature Extraction v10.7.1 with linear normalisation (protocol CGH_107_Sep09) (both from Agilent Technologies Inc.). Data analysis was performed using Nexus Copy Number software ${ }^{\circledR}$ Discovery Edition v8.0 (BioDiscovery Inc., El Segundo, CA, USA) as previously described (21). The FASST2 segmentation algorithm was used to define non-random regions of CNAs across the genome with a significance threshold set to $\mathrm{P}=1.0 \times 10^{-6}$. The $\log ^{2}$ ratio thresholds for aberration calls were set to 1.5 for high copy number gain/amplification, 0.2 for gain, -0.2 for loss, and -1.5 for homozygous deletion. Each aberration was checked manually to confirm the accuracy of the call. Sex chromosomes and regions partially or completely covered by a previously reported copy number variation (Database of Genomic Variants; http://dgvbeta.tcag.ca/dgv/app/news?ref=NCBI37/hg19) were excluded from the analysis.

Mitochondrial DNA sequencing. Whole mitochondrial genome sequencing was performed on the Ion $\mathrm{PGM}^{\mathrm{TM}}$ system with the Precision ID mtDNA Whole Genome Panel (both Thermo Fisher Scientific, Inc., Waltham, MA, USA). Library 

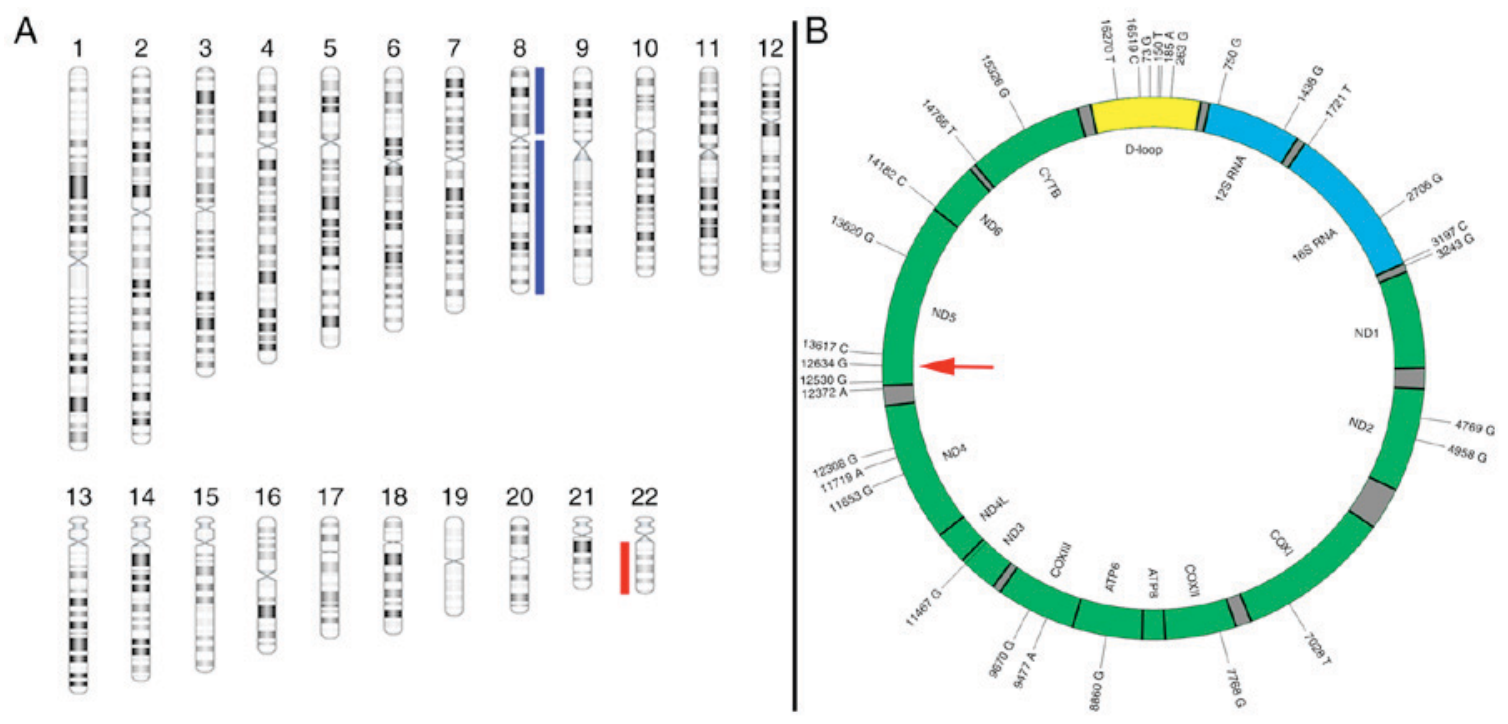

Figure 2. (A) Schematic, partial karyogram demonstrating a gain of one copy of chromosome 8 (vertical blue line) and loss of one copy of chromosome 22 (vertical red line) as the sole genomic imbalances in the lacrimal oncocytoma. (B) Sequencing of the mitochondrial DNA revealed multiple non-synonymous single nucleotide polymorphisms in genes $(N D 2, N D 5$, and $N D 6$ ) involved in the respiratory complex I. Several synonymous single nucleotide polymorphisms were identified in the entire mitochondrial genome. One possibly damaging mutation was identified in the ND5 gene (arrow). ND, NADH-ubiquinone oxidoreductase chain.

preparation was performed according to the Ion AmpliSeq Kit for Chef DL8 with minor modifications. After PCR of panel pool 1 and pool 2, the products were pooled for library preparation. Ion PGM IC 200 kit was used as the template kit for the IonChef (both from Thermo Fisher Scientific, Inc.). Sequencing was carried out using Ion PGM IC 200 Sequencing kit (TRS) with the Ion $318^{\mathrm{TM}}$ Chip v2 (Thermo Fisher Scientific, Inc.). Variant calling was carried out using the Torrent Variant Caller v4.6 of the Torrent Suite ${ }^{\mathrm{TM}}$ software (Thermo Fisher Scientific, Inc.). PrecisionID_mtDNA_rCRS.fasta was used as the reference genome with PrecisionID_mtDNA_WG_targets. bed as panel the BED (Thermo Fisher Scientific, Inc.), and PrecisionID_mtDNA_TVCv4.6_AnalysisParams.json as the analysis parameter settings (Thermo Fisher Scientific, Inc.).

Variants were discarded if the coverage was below $\mathrm{x} 25$ and the minimum heteroplasmy threshold level was set to $10 \%$ of the coverage. Data was analysed using the MitoMaster tool (22) and the MitImpact version 2.7 (23). The Polyphen-2 tool was used to predict harmful single nucleotide polymorphisms (SNP) with a frequency $<0.5 \%$ in the $\operatorname{GenBank}^{\circledR}(24)$.

\section{Results}

Histopathology. Macroscopically, the tumor measured $25 \times 22 \times 17 \mathrm{~mm}$. The tumor was non-encapsulated. The colour was deep red with blue cystic areas. Microscopic examination revealed large, cylindrical, eosinophilic and basal oriented tumor cells (Fig. 1D). It was not possible to identify normal ducts between the tumor cells, but normal ducts were located in the periphery of the specimen, possibly consistent with lacrimal gland oncocytoma, arising from a glandular duct. PAS staining was positive in a small fraction of the tumor cells and in the cystic content. Staining with Alcian blue was negative. PTAH was positive in the tumor cells. An extended immunoprofile included strong reactivity for CK 5/6, CK 7, CK 8/18, CK 17, CK 19, S-100, and EMA. CD117 was slightly positive and CK 14 was positive in $\sim 30 \%$ of the basal-type cells. The cytokeratin profile was similar to that of normal lacrimal gland tissue and other ocular adnexal oncocytomas (1). The tumor cells stained positively for the mitochondrial antigen, MU213-UC (Fig. 1E). Staining with anti-Ki67 revealed positivity in $<1 \%$ of the tumor cell nuclei, indicating a low proliferative index. The tumor cells were negative for CK 20, CEA and SMA.

Genetic profile. Genome-wide aCGH analysis revealed a genomic profile characterised by gain of one copy of chromosome 8 and loss of one copy of chromosome 22 as the sole imbalances (Fig. 2). There was no evidence of gene amplifications or homozygous deletions.

mtDNA sequencing. Sequencing of the mtDNA revealed 33 sequence variants (Table II). Five SNPs were recognised in $<0.5 \%$ of the samples in the GenBank ${ }^{\circledR}$. Four non-synonymous SNPs were identified in the NADH-ubiquinone oxidoreductase chain 5 (ND5) gene, which is part of the respiratory complex I. Additionally, an insertion at site 524 was identified in the displacement-loop (D-loop). One non-synonymous SNP (A12634G) possibly had a damaging effect. Additionally, SNPs were also identified in the ND2, ND4 and ND6 genes of the respiratory complex I. However, these SNPs were synonymous. Two non-synonymous SNPs (A9670G and G9477A) were identified in the cytochrome oxidase subunit III gene, which is part of the respiratory complex IV. None of the synonymous SNPs were involved in splice sites. No transversions were identified.

\section{Discussion}

The present study described the immunohistochemical and genetic profile of a rare case of lacrimal gland oncocytoma in a 20-year-old male. This is the 13th case of lacrimal gland 
oncocytoma in the literature. Men and women are equally affected, with a median age at the time of diagnosis of 57 years (range, 1-83 years; Table I). The symptoms of lacrimal gland oncocytoma range from mild lid swelling without pain to proptosis with severe pain $(10,13)$. The majority of lacrimal gland oncocytomas were surgically removed through a lateral orbitotomy or more rarely through a fronto-orbitozygomatic craniotomy (13). All patients with lacrimal gland oncocytoma were alive at the time of the last follow-up (3-42 months) (8). Malignant transformation or recurrence of lacrimal gland oncocytoma following complete surgical excision has not been reported. Hence, a possible association between oncocytoma and the exceedingly rare oncocytic carcinoma is unclear.

Little is known about the genetic changes leading to oncocytoma formation. In the present case, a gain of one copy of chromosome 8 and loss of one copy of chromosome 22 were identified as the sole genomic imbalances. To the best of our knowledge, none of these alterations have previously been described in oncocytoma of any sites. The significance of the findings of the current study is unclear. However, it appears that genomic instabilities are typical for oncotoma that occurs in males (16). Exome sequencing of renal oncocytoma has identified two main types. A diploid oncocytoma that has no sex predilection and another type that has a male predilection, and is a hypodiploid oncocytoma with complete loss of chromosome 1, 14, 21, X, or Y (16). Other chromosomal aberrations have been described in oncocytic lesions of the thyroid, including losses and gains of both arms of chromosomes 1, 2, 5, 7, 12, 17, 19, 20 and 22 (4). However, only gains involving chromosome 22 have been reported, contrasting with the loss of chromosome 22 in the present case (4). The trisomy 8 in the current case is similarly unprecedented, but trisomy 7 has been demonstrated in a case of salivary gland oncocytoma (25). There may be a site specific difference in the alterations identified in oncocytoma, as the frequent chromosomal aberrations observed in renal, thyroid and salivary oncocytoma are different, and diffuse (16-18,25). This supports the idea that the results of the present study may be specific to lacrimal gland oncocytoma, since these changes have not been observed elsewhere. Deletions, SNPs and rearrangements have been described in mtDNA in oncocytoma of the thyroid, kidney, salivary glands, and adrenal cortex (2). In line with the apparent association between the oncocytic phenotype and mtDNA gene aberrations, specifically in genes involved in complex I function, several sequence variants in the mitochondrial genome of the present case were identified. The majority of these were transitions, but one insertion was identified at mtDNA position 524. Insertions in the D-loop have previously been reported in thyroid adenoma (18). In addition, six SNPs were identified in $<0.5 \%$ of the 32,000 mitochondrial genomes registered in the GenBank ${ }^{\circledR}$. Notably, two synonymous and four non-synonymous SNPs were detected in the ND5 gene, which is a part of the respiratory complex I. Of note, the consequence of one SNP in ND5 was termed 'probably damaging' with the PolyPhen-2 tool for functional annotation of genetic variants, thereby supporting a fundamental pathogenic similarity between lacrimal gland oncocytoma and oncocytic lesions in other anatomical sites. Overall, these mtDNA variants may potentially impair cell respiration, and be responsible for the slow-proliferating nature of the tumor.
It is thought that mutations in the mitochondrial genes encoding proteins involved in oxidative phosphorylation may result in compensatory mitochondrial proliferation, while mutations in nuclear genes encoding oxidative phosphorylation proteins are less frequently involved (2). The cause of mitochondrial proliferation and the possible association with proliferation of the oncocyte itself remains unclear.

In conclusion, a gain of one copy of chromosome 8 and loss of one copy of chromosome 22 were identified in a rare case of lacrimal gland oncocytoma. In addition to these gross alterations of nuclear DNA, a peculiar involvement of apparently damaging mitochondrial point mutations resulting in impairment of respiratory function was reported, similar to what has previously been reported in oncocytoma from other anatomical sites, suggesting a potential involvement of mitochondria in oncocytoma pathogenesis.

Oncocytoma of the ocular adnexa is more common in the lacrimal caruncle (1). It would be of interest in future studies to compare $\mathrm{CGH}$ and mitochondrial sequencing results of a selection of these lesions to the current case to evaluate the molecular changes in ocular adnexal oncocytoma.

\section{Acknowledgements}

The present study was supported by the Swedish Cancer Society (grant no. 160509).

\section{References}

1. Østergaard J, Prause JU and Heegaard S: Oncocytic lesions of the ophthalmic region: A clinicopathological study with emphasis on cytokeratin expression. Acta Ophthalmol 89: 263-267, 2011.

2. Máximo V, Rios E and Sobrinho-Simoes M: Oncocytic lesions of the thyroid, kidney, salivary glands, adrenal cortex and parathyroid glands. Int J Surg Pathol 22: 33-36, 2014.

3. Andreasen S, Esmaeli B, Holstein SL, Mikkelsen LH, Rasmussen PK and Heegaard S: An update on tumors of the lacrimal gland. Asia Pac J Ophthalmol (Phila) 6: 159-172, 2017.

4. Gasparre G, Bonora E, Tallini G and Romeo G: Molecular features of thyroid oncocytic tumors. Mol Cell Endocrinol 321: 67-76, 2010.

5. Beskid $\mathrm{M}$ and Zarzycka $\mathrm{M}$ : A case of onkocytoma of the lacrimal gland. Klin Oczna 29: 311-315, 1959 (In Polish).

6. Riedel K, Stefani FH and Kampik A: Oncocytoma of the ocular adnexa. Klin Monatsbl Augenheilkd 182: 544-548, 1983 (In German)

7. Hartman LJ, Mourits MP and Canninga-van Dijk MR: An unusual tumour of the lacrimal gland. Br J Ophthalmol 87: 363 , 2003.

8. Calle CA, Castillo IG, Eagle RC and Daza MT: Oncocytoma of the lacrimal gland: Case report and review of the literature. Orbit 25: 243-247, 2006.

9. Archondakis S, Skagias L, Tsakiris A, Sambaziotis D and Daskalopoulou D: Oncocytoma of the lacrimal gland diagnosed initially by fine-needle aspiration cytology. Diagn Cytopathol 37: 443-445, 2009

10. Economou M, Seregard S and Sahlin S: Oncocytoma of the lacrimal gland. Acta Ophthalmologica 85: 576-577, 2007.

11. Kim JY, Park HY, Paik JS, Kim DC and Yang SW: Oncocytoma of the lacrimal gland: An Asian case. Jpn J Ophthalmol 54: 239-241, 2010

12. Aghaji AE, Olushina DB and Okoye OI: Oxyphil cell adenoma in a Nigerian: Case report and review of the literature. Niger J Clin Pract 14: 373-376, 2011.

13. Limb RJ, Rosenfeld JV and McLean C: Giant orbital oncocytoma. Asian J Neurosurg 8: 192-194, 2013.

14. Ferte A, Trechot F, Cloche V, Busby H, Maalouf T, Angioi K and George JL: Oncocytoma: An uncommon lesion of the lacrimal gland. J Fr Ophtalmol 39: e231-e233, 2016. 
15. Jittapiromsak N, Hou P, Williams MD and Chi TL: Orbital oncocytoma: Evaluation with dynamic contrast-enhanced magnetic resonance imaging using a time-signal intensity curve and positive enhancement integral images. Clin Imaging 42: 161-164, 2017.

16. Joshi S, Tolkunov D, Aviv H, Hakimi AA, Yao M, Hsieh JJ, Ganesan S, Chan CS and White E: The genomic landscape of renal oncocy toma identifies a metabolic barrier to tumorigenesis. Cell Rep 13: 1895-1908, 2015.

17. Gasparre G, Porcelli AM, Bonora E, Pennisi LF, Toller M, Iommarini L, Ghelli A, Moretti M, Betts CM, Martinelli GN, et al: Disruptive mitochondrial DNA mutations in complex I subunits are markers of oncocytic phenotype in thyroid tumors. Proc Nat Acad Sci USA 104: 9001-9006, 2007.

18. Maximo V, Lima J, Soares P, Botelho T, Gomes L and Sobrinho-Simões M: Mitochondrial D-loop instability in thyroid tumours is not a marker of malignancy. Mitochondrion 5: 333-340, 2005

19. World Medical Association: World Medical Association Declaration of Helsinki: Ethical principles for medical research involving human subjects. JAMA 310: 2191-2194, 2013.

20. Andreasen S, Persson M, Kiss K, Homøe P, Heegaard S and Stenman G: Genomic profiling of a combined large cell neuroendocrine carcinoma of the submandibular gland. Oncol Rep 35: 2177-2182, 2016
21. Persson F, Winnes M, Andrén Y, Wedell B, Dahlenfors R, Asp J, Mark J, Enlund F and Stenman G: High-resolution array CGH analysis of salivary gland tumors reveals fusion and amplification of the FGFR1 and PLAG1 genes in ring chromosomes. Oncogene 27: 3072-3080, 2008.

22. Lott MT, Leipzig JN, Derbeneva O, Xie HM, Chalkia D, Sarmady M, Procaccio V and Wallace DC: mtDNA variation and analysis using mitomap and mitomaster. Curr Protoc Bioinformatics 44: 1.23.1-16, 2013.

23. Castellana S, Rónai J and Mazza T: MitImpact: An exhaustive collection of pre-computed pathogenicity predictions of human mitochondrial non-synonymous variants. Hum Mutat 36: E2413-E2422, 2015.

24. Adzhubei I, Jordan DM and Sunyaev SR: Predicting functional effect of human missense mutations using PolyPhen-2. Curr Protoc Hum Genet: Chapter 7:Unit7.20, 2013.

25. Mark J, Dahlenfors R, Havel G and Böckmann P: Benign parotid oncocytoma with the chromosomal abnormality trisomy 7. Anticancer Res 11: 1735-1737, 1991. 